\title{
Supporting Structural and Functional Collaborative Networked Organizations Modeling with Service Entities
}

\author{
Rubén Darío Franco, Ángel Ortiz Bas, Guillermo Prats, \\ and Rosa Navarro Varela \\ CIGIP Research Centre on Production Management and Engineering, \\ Edificio 8G, Acceso D, Planta 4, \\ CP 46022, Valencia, Spain \\ \{dfranco, aortiz, gprats, ronava\} @cigip.upv.es
}

\begin{abstract}
This work focuses on the Service Entities definition as an approach that may help to support structural and functional Collaborative Networked Organizations (CNO) modeling, when VOs are engineered inside Virtual Breeding Environments Management Systems (VMS). Manbree is an undergoing development which is intended to provide an integrated framework for CNO modeling and execution based on that approach and it is briefly described at the final section.
\end{abstract}

Keywords: Collaborative Networked Organizations, Enterprise Modeling, Service Oriented Architecture, Virtual Breeding Environment Management System.

\section{Introduction}

Collaboration among geographically dispersed entities, organizations or individuals, is increasing due to the facilities given by Information and Communication Technologies (ICT). Those technologies allow Virtual Breeding Environments enhance the effectiveness and rapidness of Virtual Organizations creation and operation.

But in order to realize such vision, it is necessary to design and implement a new kind of systems which can be able of managing both VBE and VO Life Cycles in a consistent and integrated way. The so-called VBE Management Systems are expected to fully support the creation, operation and dissolution of Virtual Organizations, by defining a set of reference models (at structural, functional or behavioral level, to name few of them) that must be adopted by VBE/VO participants when they are willing to be involved in collaboration opportunities.

By adopting such VBE-MS Reference Models, VBE/VO participants agree on common engineering and operating principles. Consequently, for really achieving such VBE/VO alignment, reference modeling plays a preponderant, if no critical, role. When exist, VO Reference Models provides reliable procedures for engineering those environments and more flexible operational structures can be easily deployed.

The research questions this paper raises are: how VBE and VO reference models must be supported by a VBE-Management System? How do they really may enable 
faster VO deployments? Which kind of architectural approach may be helpful for this purpose and how participants may take advantage of it?

As we consider that model-driven approaches may help to find suitable approaches in answering such questions, along this paper the concept of Service Entities, as a modeling construct which may facilitate both structural and functional Collaborative Networked Organizations modeling, is introduced. Additionally, based on this approach, a supporting tool will be briefly described.

The paper has been structured as follows. In Section 2, a shortly introduction to basic concepts is given and motivation of this work is stated. In Section 3, Services Entities concept and its life cycle are briefly described. In Section 4, main ManBree's functionalities are shown, mostly from the structural and functional modeling of CNOs. Finally, Section 5, next steps are envisioned.

\section{Concepts and Motivation}

Collaboration between partners is a preferred way to ensure optimal resource balance and to get perdurable benefits [1]. As it has been defined, Virtual Breeding Environments [2] are intended to harmonize the preparedness level of involved organizations while, at the same time, a collaborative infrastructure is deployed in order to deal with interoperability problems at different levels: communications, data, services, processes or business [3].

In VBEs, the main idea is to restrict the number of potential participants by drawing a border to the open universe and allowing some partners to come inside. Those partners have to agree on common operating principles: business semantics, strategies or goals, distributed business processes management practices or even common ICT tools. Being inside the border reduces uncertainty between partners, basic to share information and to reach their common objectives. Rapidness and flexibility in VO preparation and launching are requirements that any VBE management system must accomplish.

\subsection{Virtual Organizations Model Driven Engineering}

Along this text, we will refer to the VO Engineering process, with a broader scope but in a similar sense as it has been used in [4] to define Enterprise Engineering and which aims to: "define, structure, design and implement [inter]enterprise operations as communication networks of business processes, which comprise all their related business knowledge, operational information, resources and organisation relations". Additionally, we argue that at VBE/VO level, such engineering process is driven by a set of reference models defined into the VBE and used/refined at VO level.

VBE Reference Models influence the VO Engineering process by providing a set of common constructs for modeling its processes, services or data. Consequently, VBE reference models will play a key role in dealing with interoperability concerns either at business, processes, services or data modeling level respectively. Complementarily, when potential VBE (or temporal VO) participants are looking for being involved into potential collaboration opportunities, as pre-requisite they must get a valid "VBE Membership". By doing so, they are implicitly agreeing on VO particular reference models (and VBE ones), depending on their intended scope. 
But we consider that there exists a decoupling point between both reference modeling approaches. In terms of structural and, mainly, functional modeling, few or none correspondence can be established between VBE and VO reference models. From the structural point of view, VBE memberships ensure that VO members can be properly identified and characterized. A registered VBE participant can be involved in several $\mathrm{VO}$ instances since it has a unique identity.

However, from the functional perspective, requirements for a VBE/VO member only appear at VO engineering level and they frequently lack of consistency among several VO instances, even if they are belonging to the same VBE. For example, a VBE member offering a single business service may be required to support as many ad-hoc interfaces as VO memberships it may have. This approach would cause most of interoperability problems to appear, mainly when those potential partners need to overcome existing semantic gaps at either process, services or data levels.

In dealing with those modeling needs, a comprehensive modeling framework, for CNOs has been proposed by Camarinha-Matos and Afsarmanesh [5]. ARCON is intended to support most of CNO modeling needs, by considering both an internal and external perspectives. From its internal perspective and covering the whole CNO lifecycle, four interrelated near-orthogonal views have been identified, namely: structural, componential, functional and behavioral dimensions.

As it has been stated earlier [6,7], proper modeling constructs are also required when engineering for both VBE and VO environments. Modeling constructs are required for any modeling approach but this is a development that still must be introduced in ARCON. Next section introduces Service Entities which, in our consideration, are a promising approach in supporting some of those needs.

\section{Service Entities (SE)}

\subsection{Concept}

Service Entities (SE) are proposed as modelling constructs for CNOs, for both the Structural and Functional dimensions. As they have been defined, a single Service Entity [8] is the result of logically tying together:

- A finite set of business services which jointly defines the expected behaviour of those conceptual entities involved into the domain being modelled and,

- A finite set of attributes which will allow characterize and distinguish between them.

Depending on the modelling scope, SEs are classified into: Abstract Service Entities (ASE) and Concrete Service Entity and (CSE).

- An Abstract Service Entity is generic building block used to represent different 'types' of entities that are present in the problem domain, mostly at VBE level. ASEs are not associated with any specific instance of entities they are defining.

They only represent the abstract definition of the attributes and also the specifications of the electronic business interfaces which are being defined for them. An example of a modelled ASE would be an abstract entity 
"bank" for which two services (interfaces) have been defined: bank account validation and account balance sheet.

- Concrete Service Entity: since they are expected to be real entities of the problem domain, CSE are instances of their corresponding ASE. Instantiating an ASE means to provide meaningful values to its attributes and specific implementation for the service interfaces (i.e. bank account validation and account balance sheet). At modelling level, this means to create an open repository where CSEs can be searched, discovered and used to create and launch VO scenarios.

\subsection{Service Entities Life Cycle}

As defined, Service Entities will also have their own lifecycle. In Figure 1, it is possible to identify some of their main activities.

At their first stage, ASE must be modelled into the VBE Engineering Environment (Figure 1, Step 1). At this stage, and as in the object orientation paradigm, ASE may be refined or generalized as needed. In this way, each specific VBE will count with a repository of ASE that will be used during the instantiation process (Figure 1, Step 2). During the instantiation process, CSE are registered in an Open Repository which can be, later on, accessed from the VO Engineering Environment. This instantiation process provides the membership applicants with the service interfaces that they ought to locally deploy and integrate at level.

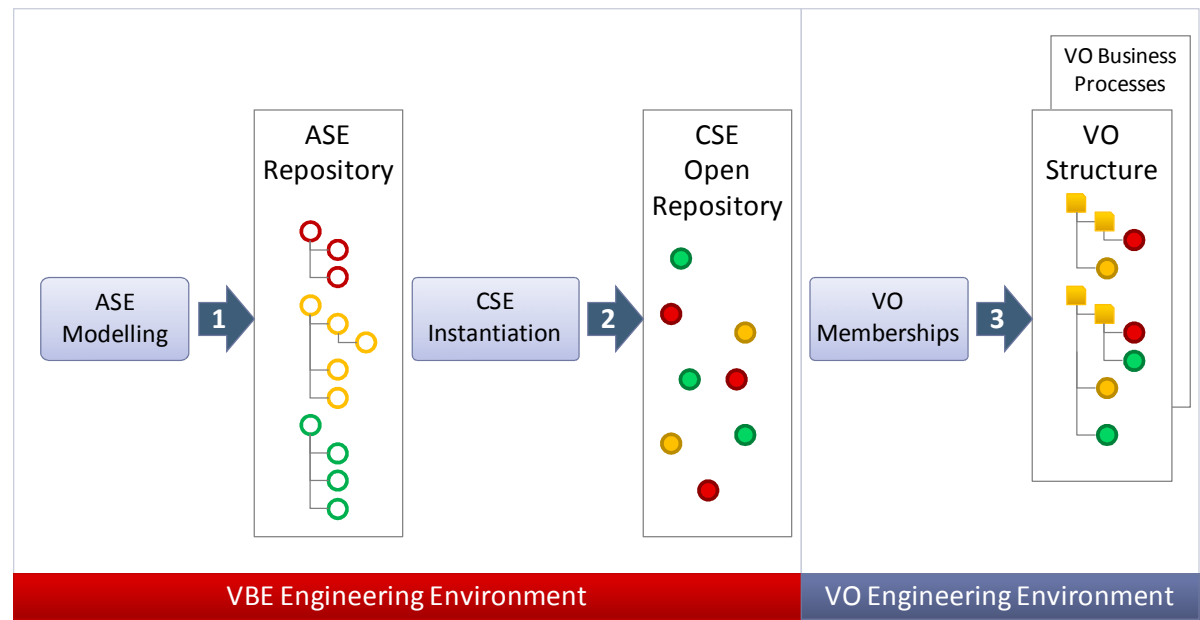

Fig. 1. Main activities of the SE lifecycle

From now on, they are prepared to be involved into as many VOs as they can be granted to be (Figure 1, step 3). The SE approach may provide some added-value for CNO modelers. To the structural dimension they provide:

- A mechanism that can be used for representing abstract VBE members' profiling and capabilities in terms of business services and interfaces. 
- VBE memberships can be supported on ASE definitions and, consequently, identity and credentials validation would run smoothly.

- VO structure may be arranged from the CSE repository and the VO Manager counts with a trusted and uniquely identified set of partners.

- VO engineering process may also include ASE definitions in order to support, for instance, dynamic negotiation and contracting.

Complementarily, from the functional dimension, SEs provide:

- A set of functional services which can be used as building blocks for VO Process Modeling.

- Each CSE can be reached by means of their homogeneous service interfaces, which may simplify the development of new VO applications.

- As they preserve the commonly agreed services interfaces, each potential VBE/VO member may decide how to link their own internally deployed systems (legacy or not) in order to provide the VO requested functionality.

\section{Manbree Modeling Environment}

ManBree is a Virtual Breeding Environment Management System where VBE, VO and SE life cycles are supported (as shown in Figure 2). During the VBE Operational phase, VO and SE life cycles may take place simultaneously while they are feeding each other: a SE may be involved in a VO as a member or even become a full VO Manager. In turn, VOs requires SEs involvement either in a static or dynamic way in order to model their structure and operations.

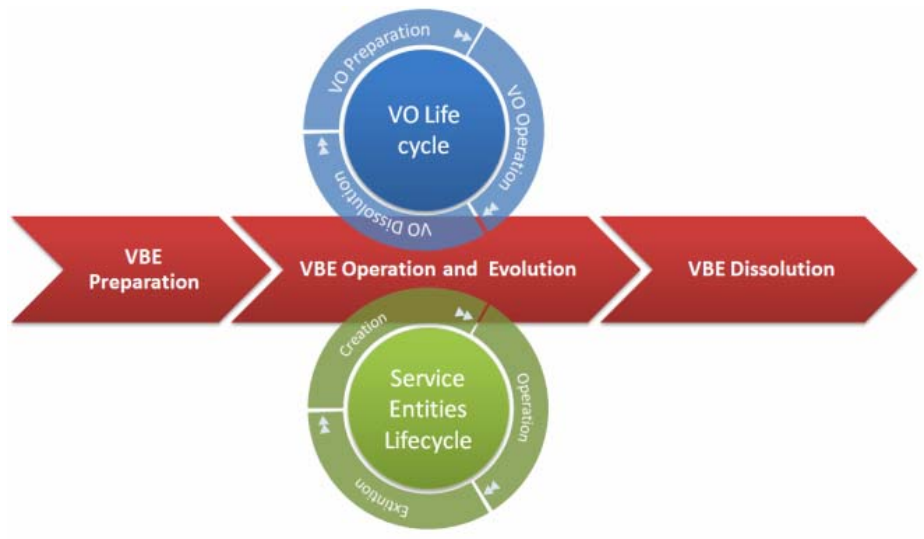

Fig. 2. ManBree Life Cycles support

\subsection{Manbree Main Functionalities}

ManBree's functionality supports three main macro-processes, namely: VBE Management, VO Management and Service Entities Management. 
In terms of VBE and VO Management functionalities, ManBree partly implements functionalities identified in Ecolead for a full VBS [9]. In Manbree implementation, a distinguishing feature is its support to the Service Entities Life Cycle, which is fully integrated both into the VBE and VO Engineering Environments by means of an Abstract Service Entities Modeling component and the Scenarios Modeling component respectively (see Figure 4, below).

In terms of Service Entities Management life cycle, supported functionalities (see Figure 3) include:

- Creation phase: At this stage, a potential Manbree member asks for its SE instantiation into a Concrete Service Entity. The instantiation procedure includes a VBE Membership application and full access to its respective ASE definition (attributes and service interfaces to be implemented)

- Operational phase: at this stage, a just created CSE may receive offers, or even apply, for different VOs, either temporary or permanently. CSE operational phase also implies: both automated and non-automated service provisioning in supporting collaborative process execution, performance measurement indicators provisioning, and knowledge management procedures, among others.

- Dissolution phase: when a CSE is going to be deleted, its performance profile is updated.

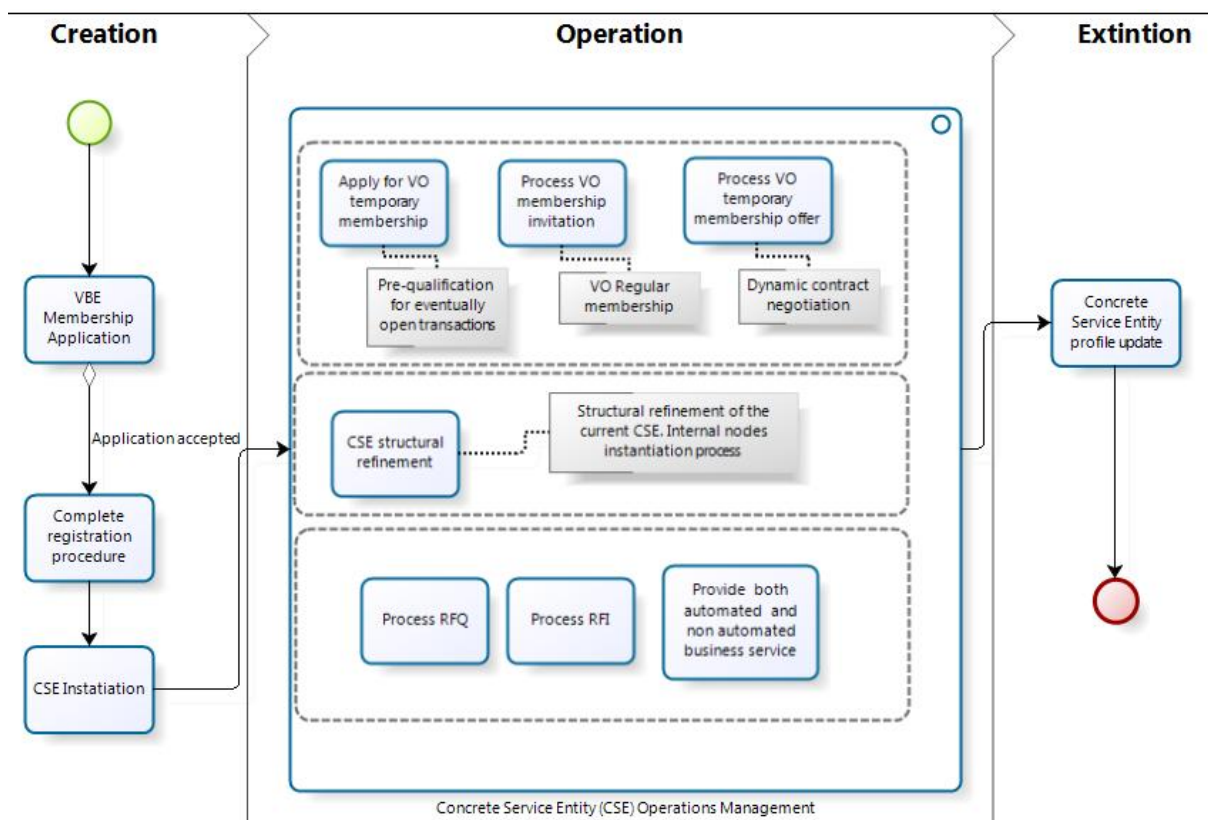

Fig. 3. Manbree Service Entities life cycle support 
Figure 4 is a screenshot of the Manbree Scenarios (VOs) Engineering component. As it can be seen, the Structural view of the scenario has been modeled by using ASE and CSE definition. The Functional View is also built up by means of previously engineered services belonging to those entities.

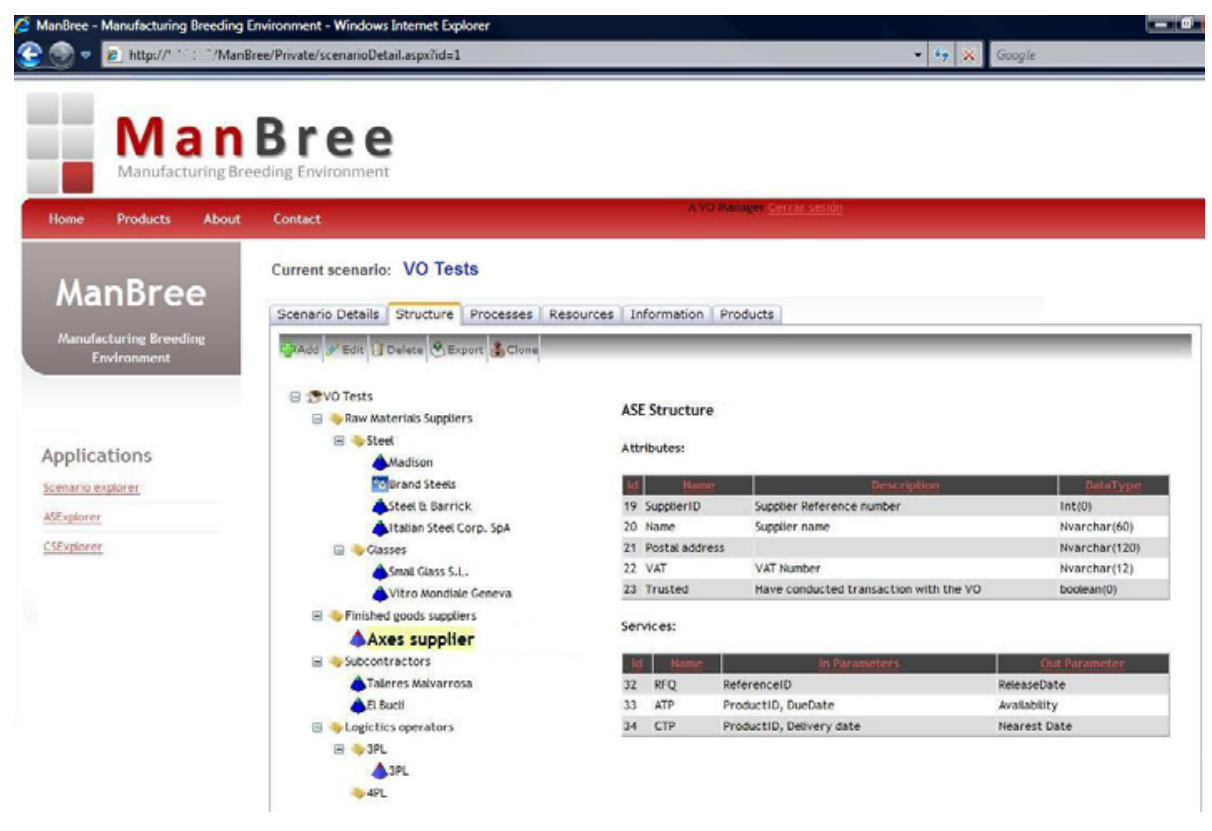

Fig. 4. SE-based Structural View of Manbree VO Engineering

\section{Conclusions}

The paper contribution has focused on introducing Service Entities as constructs that may support structural and functional CNO modeling when engineering VO inside a VBE-MS. By means of proper ASE definitions at VBE level, the instantiation procedure is able to provide concrete entities to the VO Manager. By using both ASE service definitions and CSE service implementations, the VO manager may create both structural and functional VO models.

Since this is an undergoing initiative, additional features will be incorporated into Manbree soon. Currently, VBE, VO and SE modeling needs are already being supported; next development will include evolving such VO structural and functional models into operational tools where distributed processes can be properly managed.

In doing so, Manbree currently supports collaborative processes modeling by using a XPDL-based editor which will enable us to get executable representations of VO processes to be moved into an Open Source BPMS (Business Process Management System). 


\section{References}

1. Jagdev, H.S., Thoben, K.: Anatomy of enterprise collaborations. Production Planning \& Control 12, 437-451 (2001)

2. Camarinha-Matos, L.M., Afsarmanesh, H.: Collaborative networks: a new scientific discipline. Journal of Intelligent Manufacturing 16, 439-452 (2005)

3. Ruggaber, R.: ATHENA-Advanced Technologies for Interoperability of Heterogeneous Enterprise Networks and their Applications. In: Interoperability of Enterprise Software and Applications, pp. 460-463. Springer, London (2006)

4. Kosanke, K., Vernadat, F., Zelm, M.: CIMOSA: enterprise engineering and integration. Computers in Industry 40, 83-97 (1999)

5. Camarinha-Matos, L., Afsarmanesh, H.: A comprehensive modeling framework for collaborative networked organizations. Journal of Intelligent Manufacturing 18, 529-542 (2007)

6. Ni, Q., Lu, W.F., Yarlagadda, P.K., Ming, X.: A collaborative engine for enterprise application integration. Computers in Industry 57, 640-652 (2006)

7. Kosanke, K., Vernadat, F., Zelm, M.: CIMOSA: enterprise engineering and integration. Computers in Industry 40, 83-97 (1999)

8. Franco, R., Ortiz Bas, Á., Lario Esteban, F.: Modeling extended manufacturing processes with service-oriented entities. Service Business 3, 31-50 (2009)

9. Ecolead, D21.1 Characterization of Key Components, Features and Operating Principles of the Virtual Breeding Environments (2005),

http: / /www.ve-forum.org/projects / 284/Deliverables /

d21_1_vbe.pdf 